\title{
Vascular calcifications as a marker of increased cardiovascular risk: A meta-analysis
}

\author{
R J MW Rennenberg' \\ A G H Kessels ${ }^{2}$ \\ L J Schurgers ${ }^{3}$ \\ J MA van Engelshoven ${ }^{4}$ \\ PW de Leeuw'

\section{A A Kroon'}

'Department of Internal Medicine; ${ }^{2}$ Department of Clinical Epidemiology and Medical Technology Assessment;

${ }^{3}$ Department of Biochemistry (VitaK); ${ }^{4}$ Department of Radiology, University Hospital Maastricht and Cardiovascular Research Institute Maastricht (CARIM), The Netherlands

Correspondence: R J MW Rennenberg Department of Internal Medicine, University Hospital Maastricht, P. Debyelaan 25, 6229 HX, Maastricht,

The Netherlands

Tel +3। 433877005

$\mathrm{Fax}+3$ I 433875006

Email r.rennenberg@mumc.nl

\begin{abstract}
Background: Several imaging techniques may reveal calcification of the arterial wall or cardiac valves. Many studies indicate that the risk for cardiovascular disease is increased when calcification is present. Recent meta-analyses on coronary calcification and cardiovascular risk may be confounded by indication. Therefore, this meta-analysis was performed with extensive subgroup analysis to assess the overall cardiovascular risk of finding calcification in any arterial wall or cardiac valve when using different imaging techniques.
\end{abstract}

Methods and results: A meta-analysis of prospective studies reporting calcifications and cardiovascular end-points was performed. Thirty articles were selected. The overall odds ratios (95\% confidence interval [CI]) for calcifications versus no calcifications in 218,080 subjects after a mean follow-up of 10.1 years amounted to 4.62 (CI 2.24 to 9.53 ) for all cause mortality, 3.94 (CI 2.39 to 6.50 ) for cardiovascular mortality, 3.74 (CI 2.56 to 5.45) for coronary events, 2.21 (CI 1.81 to 2.69) for stroke, and 3.41 (CI 2.71 to 4.30 ) for any cardiovascular event. Heterogeneity was largely explained by length of follow up and sort of imaging technique. Subgroup analysis of patients with end stage renal disease revealed a much higher odds ratio for any event of 6.22 (CI 2.73 to 14.14).

Conclusion: The presence of calcification in any arterial wall is associated with a $3-4$-fold higher risk for mortality and cardiovascular events. Interpretation of the pooled estimates has to be done with caution because of heterogeneity across studies.

Keywords: calcification, cardiovascular risk, meta-analysis, imaging

\section{Introduction}

Several imaging techniques may reveal vascular calcium deposits. Since these deposits are not always the prime reason for the investigation, the presence of calcified blood vessel walls or heart valves is often not recognized as a clinically important sign of vascular risk. Although calcifications may occur in small amounts in the earlier stages of atherosclerosis, they are usually seen in more advanced lesions. ${ }^{1}$ Indeed, various studies have observed an increased cardiovascular risk in patients with calcification. ${ }^{2-8}$ However, the latter is not a uniform finding. ${ }^{9}$ This discrepancy may be due to differences in methodology, patient selection and baseline risk. Recent meta-analyses of the relationship between calcification and vascular risk have focused exclusively on coronary calcium scores. ${ }^{10}$ As patients with coronary insufficiency are more likely to be subjected to diagnostic imaging than others, part of the relationship between coronary calcification and outcome may be confounded by indication. To overcome this problem, we performed the present meta-analysis in which we assessed the impact of calcium deposits in any artery or heart valve, with different imaging techniques, on fatal and nonfatal cardiovascular events as well as total mortality in populations with different baseline risks.

\section{Methods}

To identify studies on vascular calcification (mineralization detected in any part of the blood vessel wall or heart valves) and cardiovascular risk, a PubMed, Embase, and 
Cochrane library search was performed using the following search terms: "plain radiography or chest X-ray or abdominal $\mathrm{X}$-ray or mammography or panoramic radiography or ultrasound (US) or computer tomography (CT) or magnetic resonance imaging (MRI)" and (cardiovascular risk or calcification). Two investigators (RJMW Rennenberg and AA Kroon) screened all abstracts and, after applying the criteria for eligibility, decided on whether or not the full papers should be retrieved. All authors reviewed qualifying papers.

\section{Study eligibility}

Only prospective cohort studies were eligible. Paediatric studies were excluded. Articles published between 1970 and June 2008, with prospective data on human vascular calcification and well-documented end-points were selected. Case reports were excluded. Data on events in both groups, with and without calcification, had to be reported separately. To be selected for the analysis absolute numbers, ie, a two-by-two table, should be extractable from the text. References found in the selected papers that fulfilled these criteria were also selected. Only papers published in English, German, Dutch, and French were considered. When data were published in consecutive articles by the same authors only the article with the most comprehensive dataset was considered. ${ }^{8,11-13}$

\section{Definition of end-points}

End-points were defined as all cause mortality, cardiovascular mortality (death from any cardiovascular cause), coronary event (myocardial infarction, coronary mortality, percutaneous coronary angioplasty or coronary artery bypass operation), stroke (ischemic and hemorrhagic) and any cardiovascular event (cardiovascular mortality, coronary event, stroke, peripheral revascularisation procedures or amputation, carotid endarterectomy).

\section{Statistical analysis}

Studies reporting data suitable to make two by two tables of calcification in relation to events were used to compute odds ratio's using a random effects meta-analysis. Because we expected significant heterogeneity, a meta-regression analysis with a random effect model was performed, as suggested by Egger and colleagues, ${ }^{14}$ taking into account the following variables: duration of follow up, type of imaging technique (plain radiograph, CT, or US) and baseline risk of the population, estimated as low (asymptomatic without known cardiovascular risk factors), intermediate (asymptomatic with one or more cardiovascular risk factors or diabetes but no renal insufficiency) and high (symptomatic or renal insufficiency). Publication bias was assessed with a funnel plot of the risk for any cardiovascular event. Statistical calculations were done using the STATA statistical program version 8.2 (StataCorp, College Station, TX), with the latest updates.

\section{Results}

We originally retrieved over 2,500 articles. Screening of abstracts and exclusion of case-reports resulted in 91 remaining original studies on the association between human vascular or valvular calcifications and cardiovascular risk. After applying the eligibility criteria 61 studies were excluded mainly because they were not prospective follow up studies. Only a few studies were excluded because absolute numbers could not be extracted or results were not reported as calcification versus no calcification. The remaining 30 studies, which are summarized in Table 1, could be included. ${ }^{3,5-9,13,15-37}$ Ten studies concerned plain radiography, such as mammograms, chest X-rays or X-rays of the peripheral blood vessels (eg, the femoral artery); ten studies had applied CT, mostly of the coronaries, and in 10 studies US had been used, predominantly of the heart valves. On plain radiographs vascular calcifications were defined as linear densities in the blood vessel area. With CT vascular calcification was defined as any vessel part with a density of 130 Hounsfield units or more. In the US studies highly echogenic plaques producing bright white echoes with shadowing were noted as calcifications. No reports with respect to MRI fulfilled the inclusion criteria. In these 30 papers, the absolute numbers of events in relation to the presence or absence of calcifications were reported, comprising a total of 218,080 subjects. Overall, $53 \%$ of the subjects were males. The prevalence of vascular calcifications varied according to the patient group studied. The lowest prevalence of $2.3 \%$ was found in an asymptomatic population with a mean age of 47 years in which aortic arch calcifications on plain chest X-rays were studied. ${ }^{5}$ The highest prevalence of $88.9 \%$ was found in patients (mean age 56 years) with chest pain in whom coronary calcifications were measured by electron beam computer tomography (EBCT). ${ }^{24}$ The median prevalence of calcification in all studies was $57.5 \%$. The mean follow up to record the number of cardiovascular events in these studies was 10.1 years (range 2-26).

\section{All cause mortality}

In nine studies, with a total of 3,821 deaths among the 40,747 participants, data on all cause mortality were re ported. ${ }^{11,17,19,22,27,28,33,36,37}$ Odds ratios are depicted in Figure 1. 


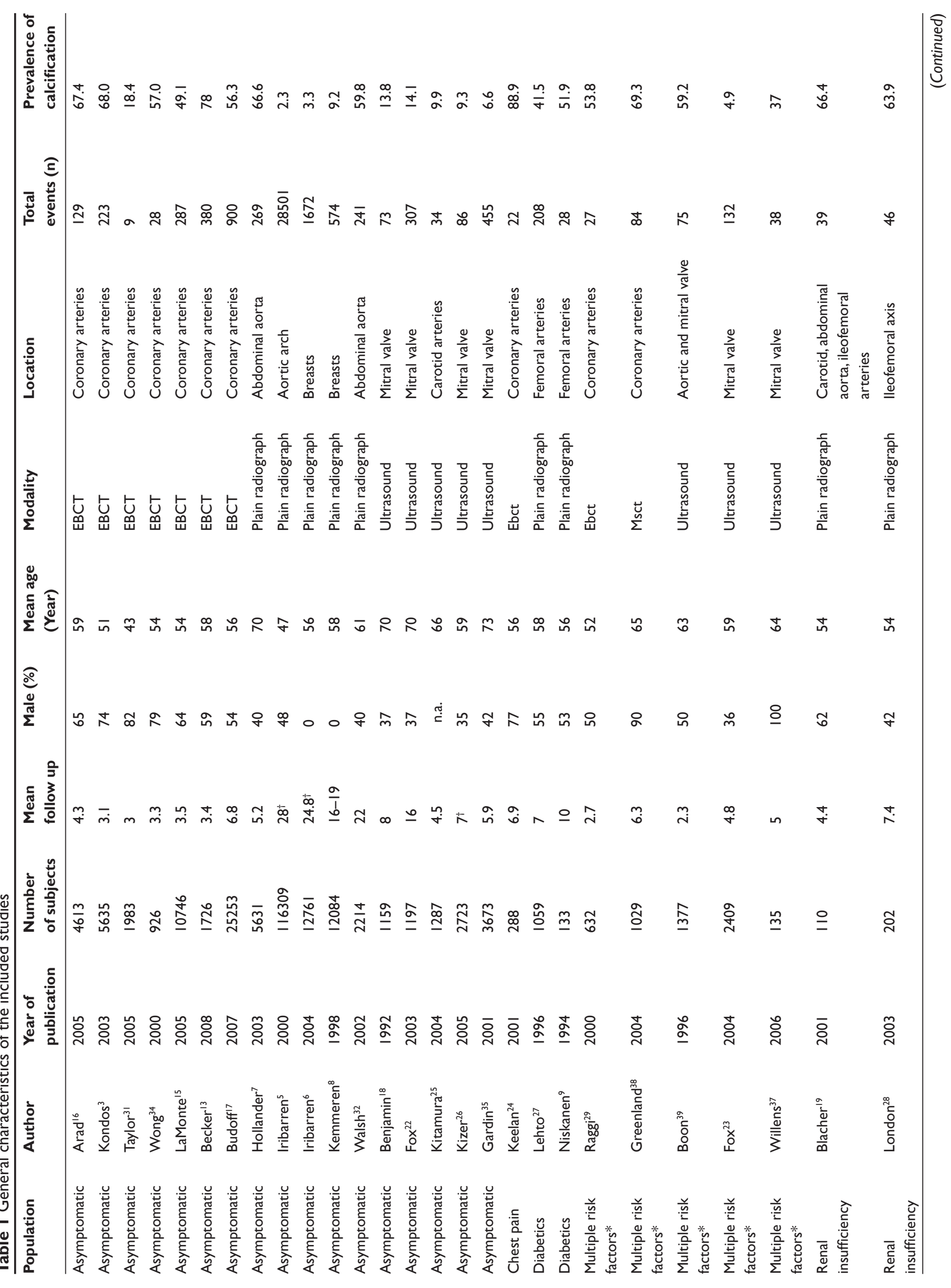




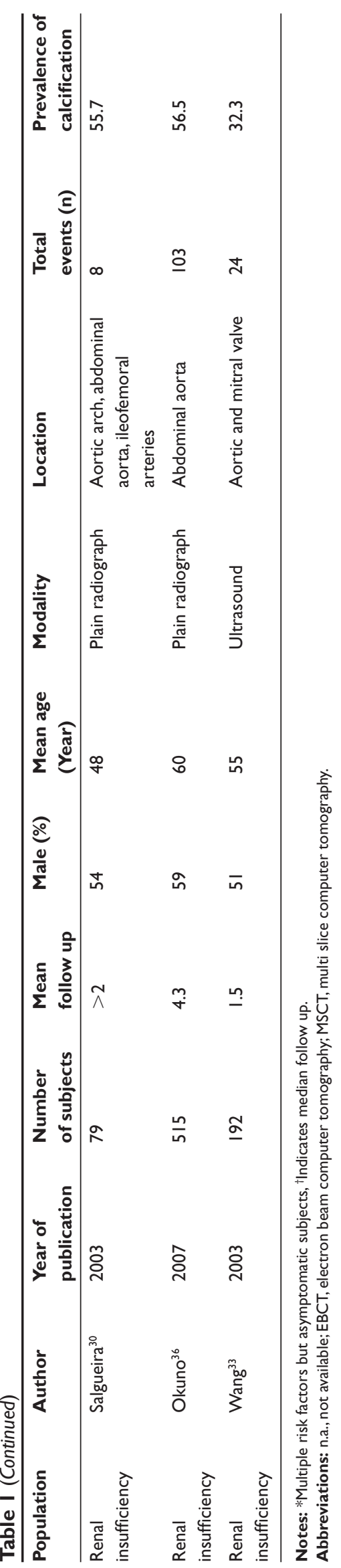

Among 16,528 subjects with calcifications 1,589 deaths were observed. Four of the studies comprised a population with renal insufficiency in which the event rate for all cause mortality in patients with calcifications was more than 5 times higher than in patients without calcifications. Overall OR for mortality from all causes was 4.62 (95\% CI 2.24 to 9.53 , Figure 1). The odds ratio for all cause mortality in patients without renal insufficiency was 3.43 (95\% CI 1.26 to 9.32 ).

\section{Cardiovascular mortality}

Odds ratios for cardiovascular mortality are also shown in Figure 1. A total of 1,356 cardiovascular deaths were observed among the 17,187 participants in the 9 studies that reported data on this complication. ${ }^{8,9,13,22,27,28,30,33,36}$ Among 3,657 subjects with calcifications, 718 cardiovascular deaths were observed. In two of the nine studies the imaging technique was US, one study employed CT, and in the remainder plain radiographs were used. The overall OR for cardiovascular mortality in the presence of calcifications was 3.94 (95\% CI 2.39 to 6.50 ), but there was significant heterogeneity among the studies (Figure 1).

\section{Coronary events}

Data on coronary events were available in 16 studies (17,482 events among 173,650 subjects). $3,5,6,8,15,16,22-24,28,29,31,33-35,38$ In the 19,261 subjects with calcifications, 1,534 events were observed resulting in an overall OR of 3.74 (95\% CI 2.56 to 5.45; Figure 2). Eight of these studies had utilized CT-scans of the coronaries and four used plain radiography. Thirteen studies originated from a North American population and most of the studies were done for screening purposes in an asymptomatic population. Average follow up in the CT-studies was only four years, but 18.7 years in the studies done with plain radiography.

\section{Stroke}

Stroke was reported as primary outcome in 11 studies with a total of 154,784 subjects of whom 9,889 showed calcifications. ${ }^{5-8,18,25-28,33,39}$ In the group with calcifications there were 874 stroke cases. The total number of events was 10,454 . Only one out of five studies with ultrasound looked at calcifications of the carotid artery, whereas in the other four US of the heart valves was performed. The remaining six studies, all with plain radiographs, evaluated vascular calcifications of the breast, the thoracic or abdominal aorta and peripheral arteries. None of the studies with $\mathrm{CT}$ assessed the incidence of stroke. The overall OR for stroke was 


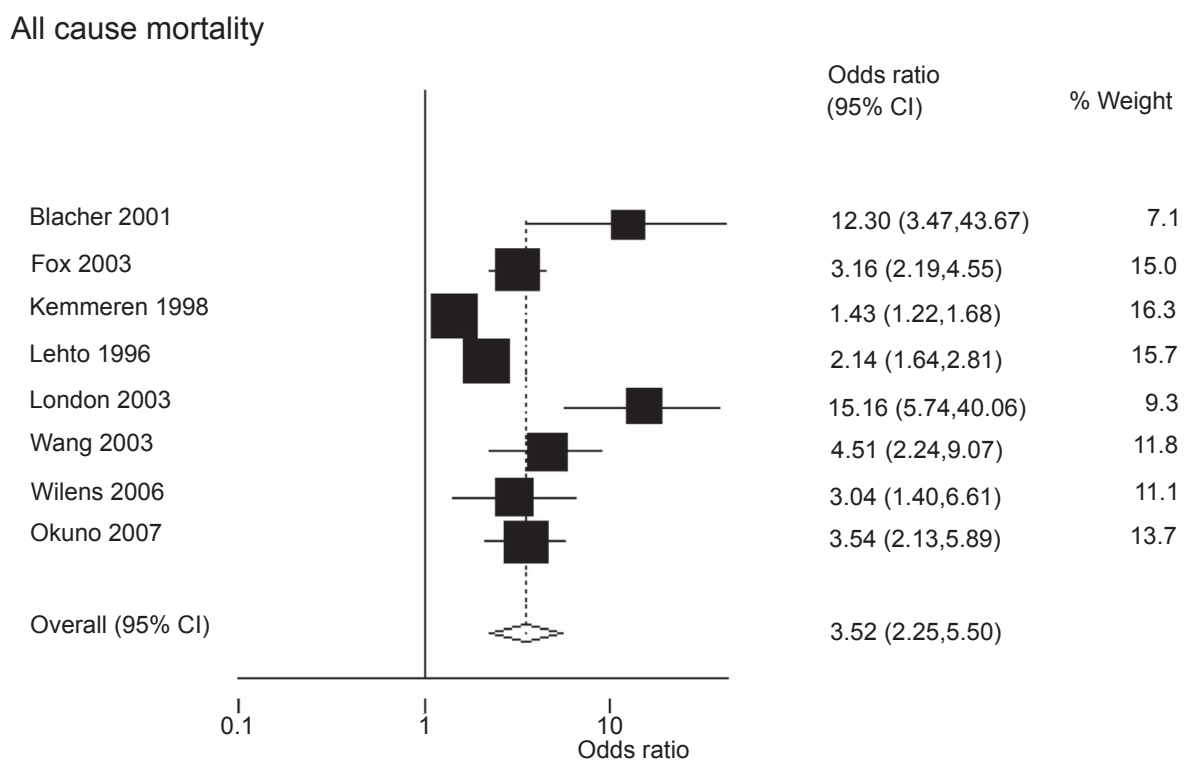

Cardiovascular mortality

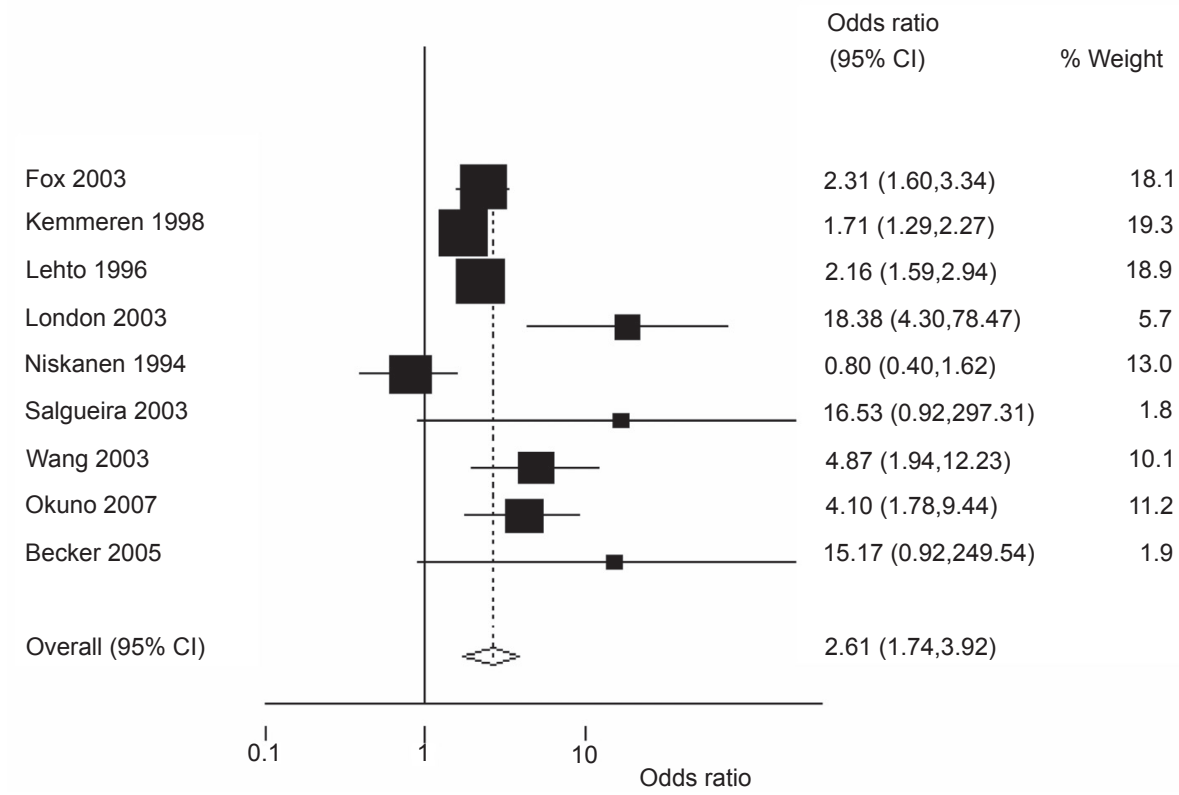

Figure I Odds ratios for mortality, all cause and cardiovascular, when calcification is present.

Notes: Heterogeneity for all cause mortality chi-squared $=248.98($ d.f. $=8) \mathrm{p}=0.000$; Heterogeneity for cardiovascular mortality chi-squared $=6 \mathrm{I} .52($ d.f. $=8) \mathrm{p}=0.000$.

2.21 (95\% CI 1.81 to 2.69 ) in the presence of calcifications anywhere (Figure 2).

\section{Any cardiovascular event}

In 27 studies with a total of 192,205 patients and 34,958 events, the risk for any cardiovascular event could be studied (Figure 3). ${ }^{3,5-9,12,15,16,18,22-36,38,39}$ In the 27,921 subjects with calcifications, 3,245 events were recorded. The average age was 59 years, but in the studies that reported the average age for the subjects with and without calcifications separately, age was significantly higher in those with calcifications
(63 versus 56 years, $\mathrm{p}<0.05$ ). The overall OR for any cardiovascular event with any modality was 3.41 (95\% CI 2.71 to 4.30). This could be subdivided in an OR of 2.14 (95\% CI 1.65 to 2.77) for ultrasound studies, 3.01 (95\% CI 2.16 to 4.20) for plain radiographic studies, and 7.26 (95\% CI 4.42 to 11.92) for studies using $\mathrm{CT}$ as diagnostic modality.

\section{Meta regression analysis for any cardiovascular event}

Meta-regression analysis with respect to the outcome of any cardiovascular event showed that the type of imaging 


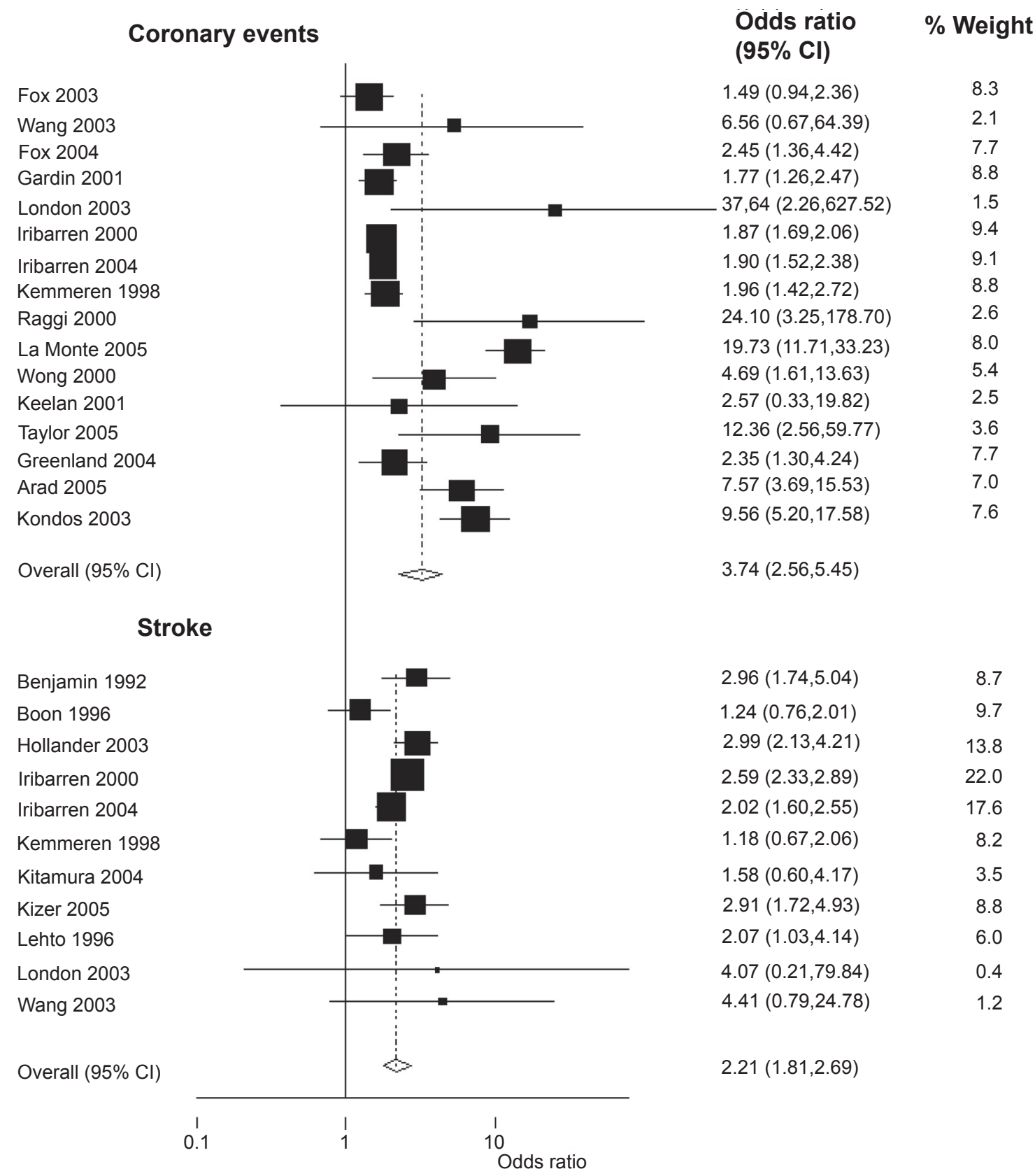

Figure 2 Odds ratios for coronary events and stroke, when calcification is present.

Notes: Heterogeneity for coronary events chi-squared $=154.86($ d.f. $=15) p=0.000$; Heterogeneity for stroke chi-squared $=21.67($ d.f. $=10) p=0.017$.

technique and the duration of follow up were significant causes of heterogeneity. After stratifying for imaging technique and follow up, the meta-analysis showed substantially lesser or no significant heterogeneity. Figure 4 is shown as an example for the CT studies. However, the number of studies per group was also much smaller giving rise to less chance for heterogeneity.

When data of high risk populations or populations with renal insufficiency or diabetes mellitus were analyzed separately there was also no significant heterogeneity (chi-squared $=1.52$ [d.f. $=4] \mathrm{p}=0.823,3.59$ [d.f. $=3] \mathrm{p}=0.309$ and 0.98 [d.f. $=1]$ $\mathrm{p}=0.322$, respectively) (Figures 5,6).

\section{Subgroup analysis}

Most studies have been performed in asymptomatic populations participating in a screening program. In some, however, the indication for the test was the presence of one or more cardiovascular risk factors. The pooled OR for any cardiovascular event was 3.34 (95\% CI 2.51 to 4.43 ) in studies with low baseline risk, ${ }^{3,5-8,15,16,18,22,23,25,26,31,32,35} 3.65$ (95\% CI 1.87 to 7.12 ) 


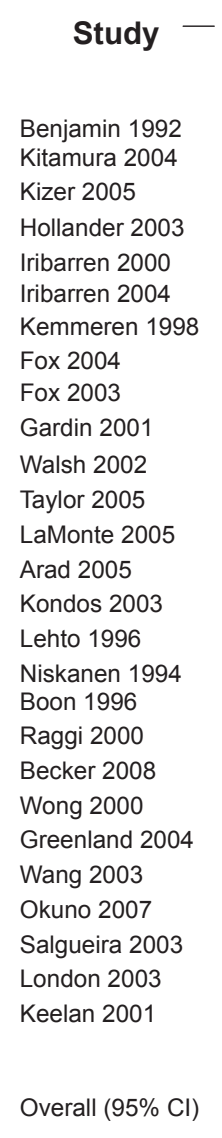

Odds ratio

(95\% Cl)

$2.96(1.74,5.04)$

$1.58(0.60,4.17)$

$2.91(1.72,4.93)$

$2.99(2.13,4.21)$

$2.47(2.28,2.67)$

$6.60(5.36,8.12)$

$1.71(1.29,2.27)$

$2.45(1.36,4.42)$

$1.69(1.19,2.39)$

$2.01(1.33,3.03)$

$2.76(2.00,3.80)$

$12.36(2.56,59.77)$

$11.08(5.10,24.07)$

$7.57(3.69,15.53)$

$9.56(5.20,17.58)$

$2.16(1.59,2.94)$

$3.56(1.39,9.10)$

$1.24(0.76,2.01)$

$24.10(3.25,178.70)$

$11.50(6.39,20.68)$

$4.69(1.61,13.63)$

$2.35(1.30,4.24)$

$4.87(1.94,12.23)$

$4.10(1.78,9.44)$

$16.53(0.92,297.31)$

$2.66(0.56,12.67)$

$2.57(0.33,19.82)$

$3.41(2.71,4.30)$
$\%$ Weight

4.4

2.9

4.4

5.1

5.7

5.5

5.3

4.2

5.1

4.9

5.2

1.6

3.5

3.7

4.1

5.2

3.0

4.6

1.1

4.2

2.6

4.2

3.0

3.3

0.6

1.6

1.0

Odds ratio

Figure 3 Risk for any cardiovascular event when calcification is present.

Notes: Heterogeneity chi-squared $=198.94($ d.f. $=26) p=0.000$.

for populations with intermediate risk ${ }^{9,13,27,29,34,38,39}$ and 4.20 ( $95 \%$ CI 2.44 to 7.23 ) in high risk subjects (Figure 5). ${ }^{24,28,30,33,36}$ There was no significant heterogeneity in the latter group for the pooled estimate.

When only subjects with renal insufficiency were considered the risk for any cardiovascular event in the presence of calcifications was even higher (Figure 6) ${ }^{19,28,30,33,36}$ All patients were on hemodialysis except for the patients in one study who were on continuous ambulatory peritoneal dialysis. ${ }^{33}$ In these five studies, comprising a total of 1098 persons, 124 events were observed. Among the 599 patients with calcifications, 104 had suffered an event. Odds ratios for any cardiovascular event were 6.22 (95\% CI 2.73 to 14.14). This pooled estimate was without significant heterogeneity. Although the confidence intervals overlap, it seemed that the presence of calcifications had the highest predictive power for a cardiovascular or a cerebrovascular event in subjects with renal insufficiency.
Although three studies reported data from diabetic patients, only two of these gave sufficient information to calculate odds ratios for cardiovascular mortality $(2.27,95 \%$ CI 1.70 to 3.04$).{ }^{9,27}$ Of 1,192 patients, 508 had calcifications on plain radiography of the femoral arteries of which 139 of a total of 236 events occurred in the patients with calcifications.

\section{Funnel plot}

A funnel plot of the standard error of log odds ratio was performed to assess a possible publication bias (Figure 7). This is based on the fact that the precision in the estimation, of the underlying effect of finding calcification on outcome, will increase as the sample size of component studies increases. Effect estimates from small studies will therefore scatter more widely at the right of the graph, with the spread narrowing among larger studies to the left of the graph. In the absence 


\section{CT only}

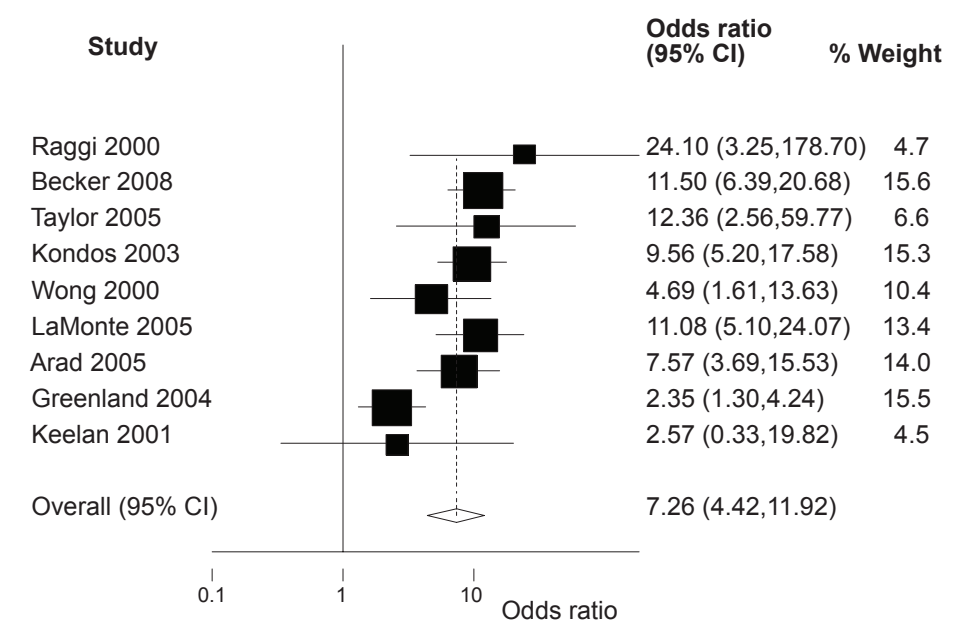

\section{CT and follow up shorter than 5 years}

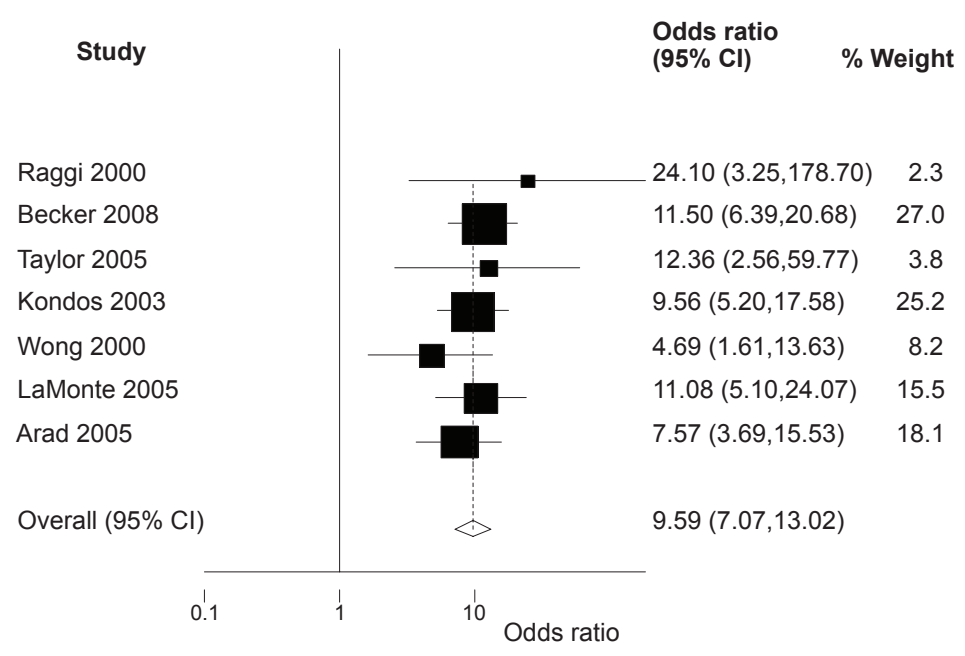

\section{$\mathrm{CT}$ and follow up longer than $\mathbf{5}$ years}

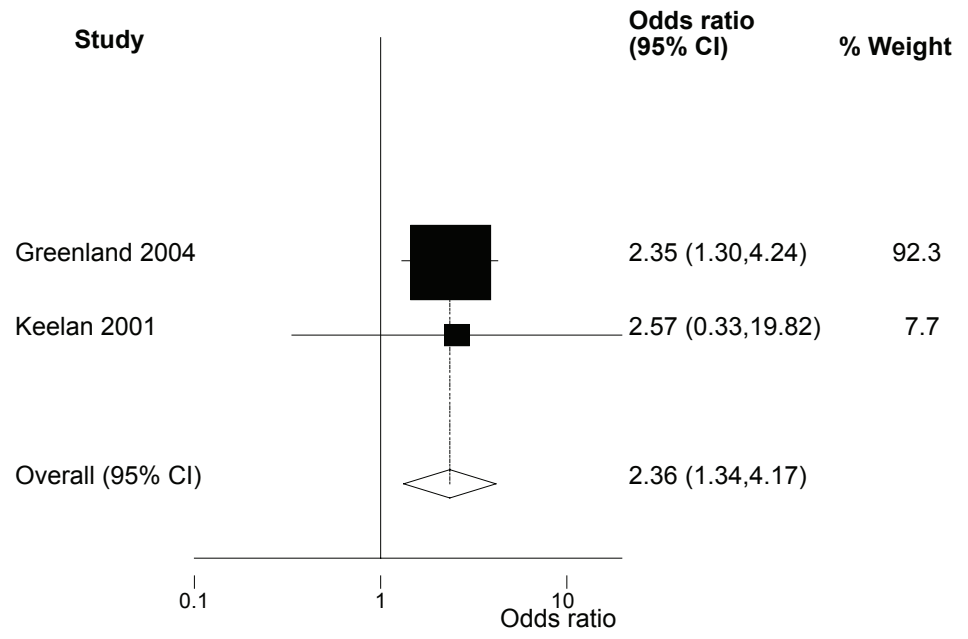

Figure 4 Results for any cardiovascular event stratified for imaging modality and follow up.

Notes: Heterogeneity chi-squared in only CT studies $=22.32(\mathrm{~d} . \mathrm{f} .=8) \mathrm{p}=0.004$; Heterogeneity chi-squared in CT studies with follow up shorter than 5 years $=3.57(\mathrm{~d} . \mathrm{f} .=6)$ $\mathrm{P}=0.735$; Heterogeneity chi-squared in CT studies with follow up longer than 5 years $=0.0 \mathrm{I}(\mathrm{d} . \mathrm{f} .=\mathrm{I}) \mathrm{P}=0.934$. 


\section{Low risk}

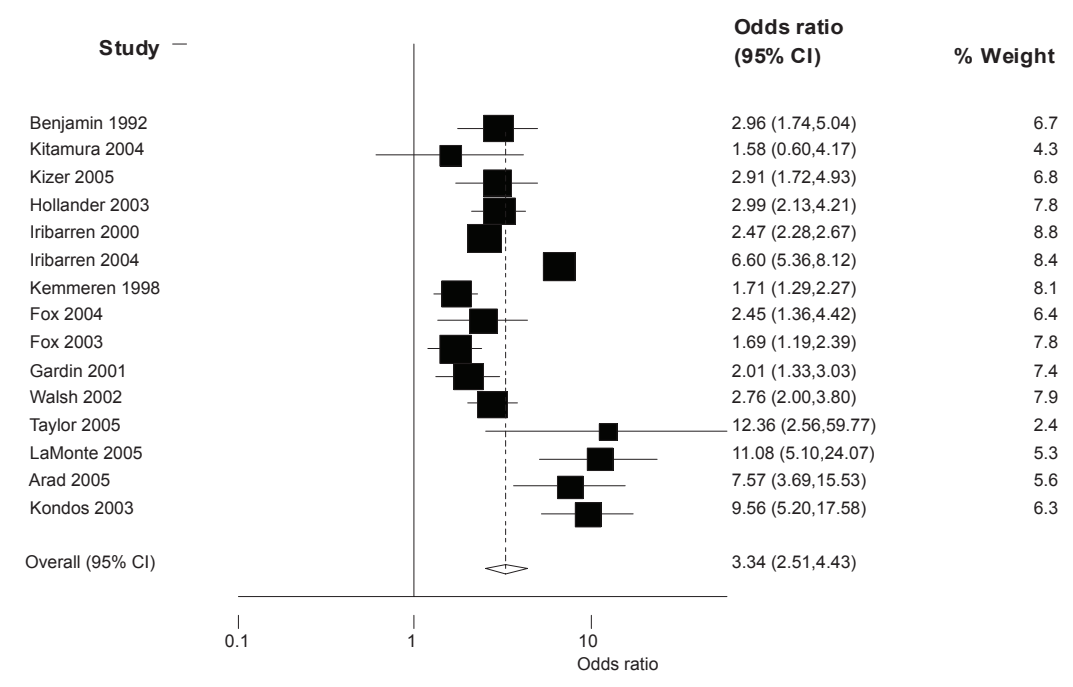

Intermediate risk

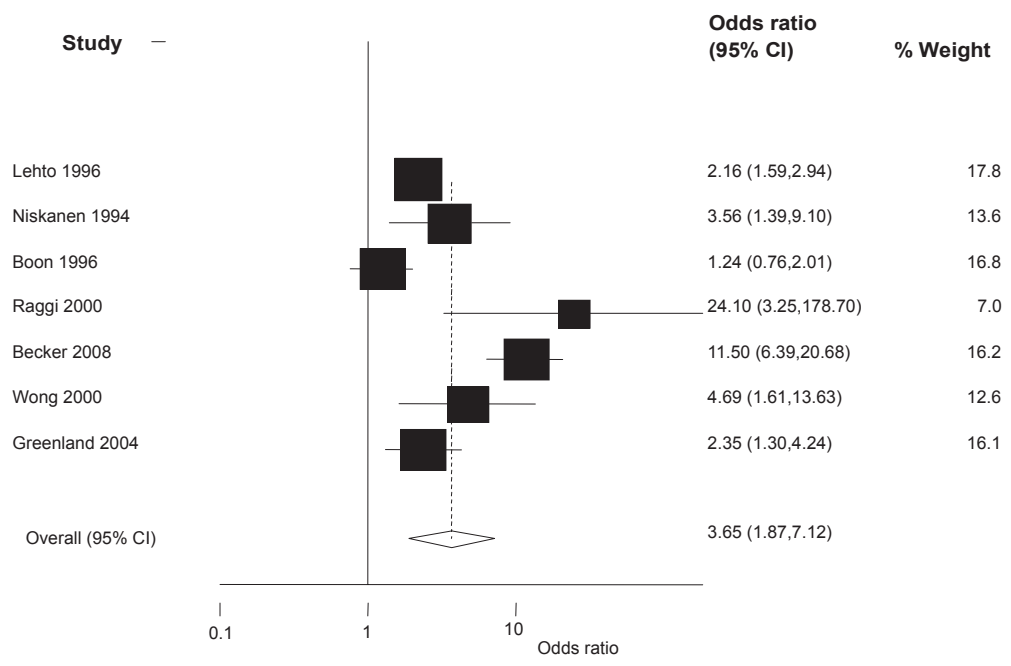

High risk

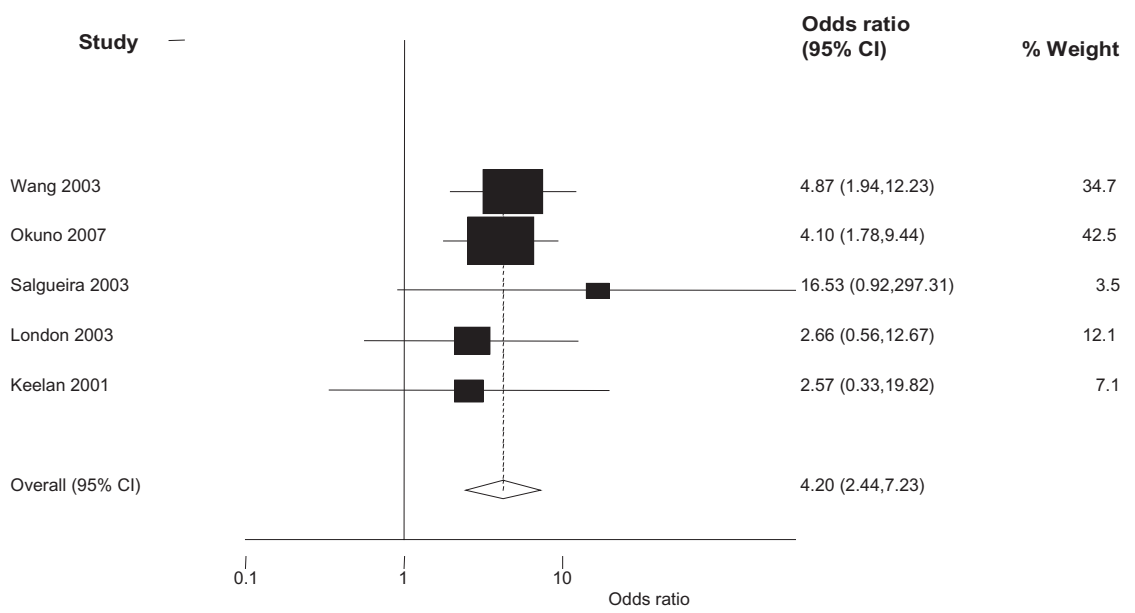

Figure 5 Risk for any cardiovascular event due to calcification according to baseline risk.

Notes: Heterogeneity in the low risk group chi-squared $=139.74($ d.f. $=14) p=0.000 ;$ Heterogeneity in the intermediate risk group chi-squared $=55.79($ d.f. $=6) \mathrm{P}=0.000$; Heterogeneity in the high risk group chi-squared $=1.52($ d.f. $=4) p=0.823$. 


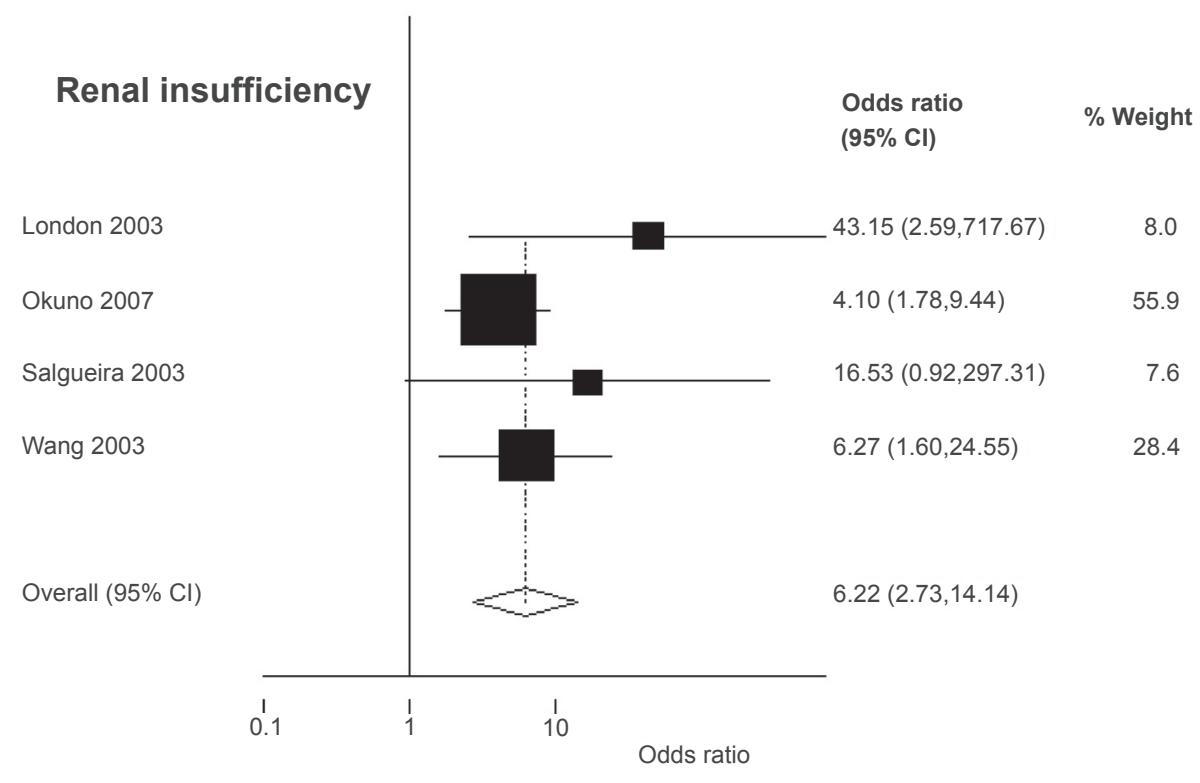

Diabetes mellitus

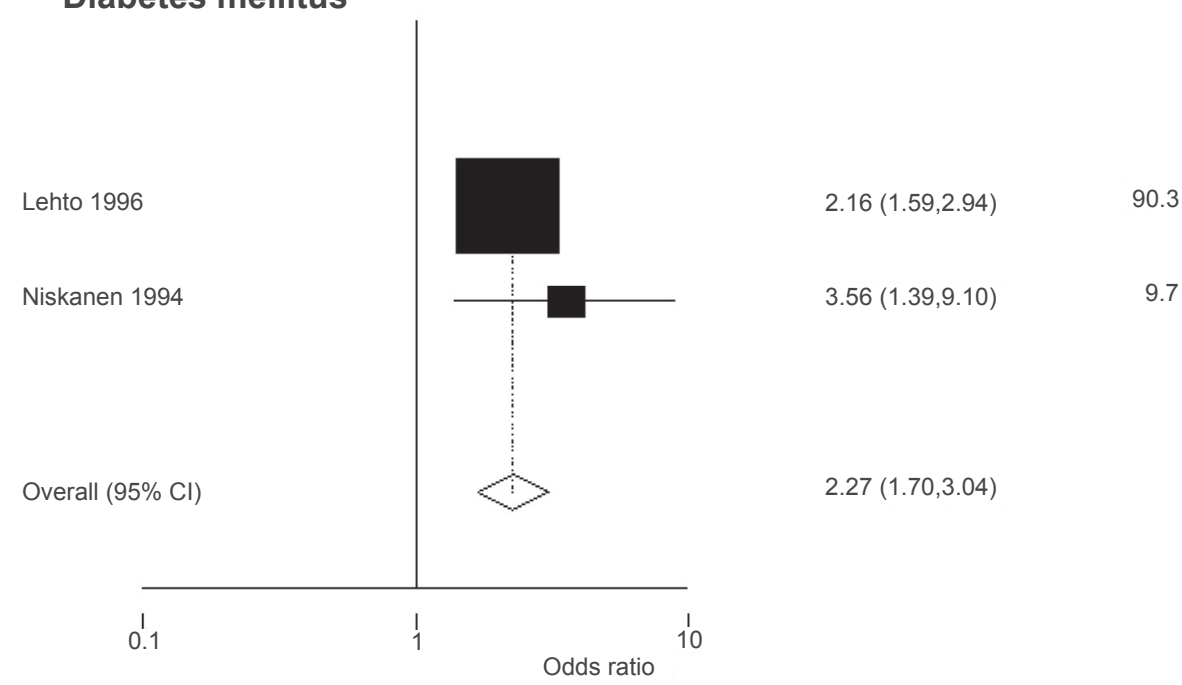

Figure 6 Subgroup analysis for renal insufficiency and diabetes mellitus.

Notes: Heterogeneity in the renal insufficiency group chi-squared $=3.59($ d.f. $=3) \mathrm{P}=0.309$; Heterogeneity in the diabetes mellitus group chi-squared $=0.98(\mathrm{~d} . \mathrm{f} .=\mathrm{I})$ $\mathrm{P}=0.322$.

of bias, the plot would appear to be symmetrical. Although it appears to be rather symmetrical, there are no studies in the right lower quadrant suggesting publication bias of small studies without any or a positive effect of calcification on cardiovascular events. However there was no bias using statistical tests for bias such as the Egger (weighted regression) method ( $\mathrm{p}$ for bias 0.24 ) or the Begg (rank correlation) method. ${ }^{14}$

\section{Discussion}

The objective of this meta-analysis was to assess the risks associated with calcification of the arterial wall or cardiac valves, as observed with different imaging methods in populations with different baseline risk. We showed that the presence of calcification with any type of imaging technique in these populations harbors a 3-4 times increased risk for mortality and cardiovascular events.

The present meta-analysis has several limitations. Firstly, it was not possible to adjust for age in our analysis, since age was not provided in all studies. Although calcification becomes more prevalent with age, we had to use unadjusted data to assess the general risk of calcification as seen on any mode of investigation. However, in almost all individual studies calcification was an independent risk factor. Adjustment for age did not change their outcome 


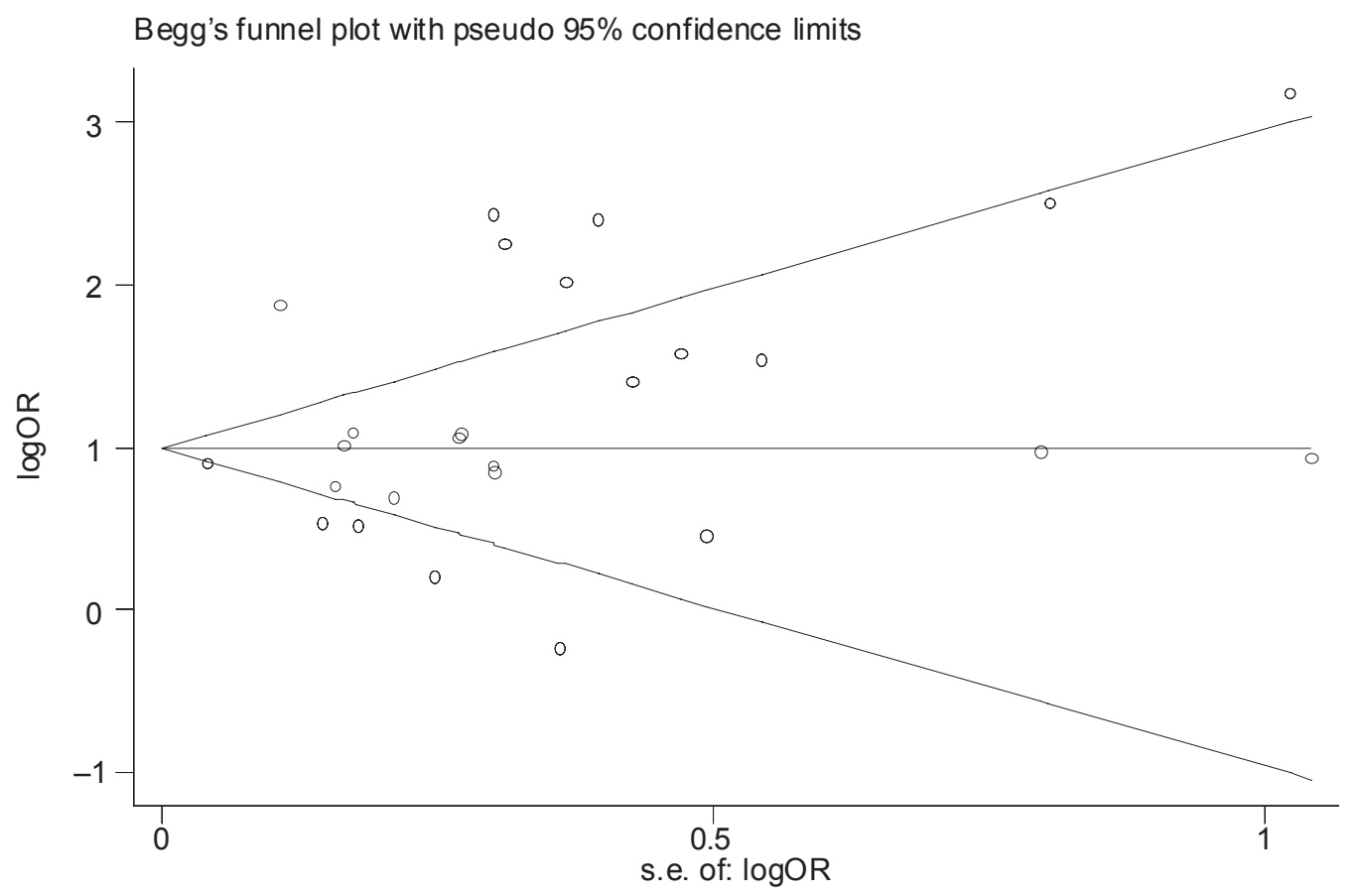

Figure 7 Funnel plot of standard error of $\log (\mathrm{OR})$ for any cardiovascular event.

Notes: Begg's funnel plot of published studies reporting any cardiovascular event. The fact that many studies are outside the $95 \%$ confidence interval, indicated by the sloping lines, is caused by heterogeneity of the studies.

substantially. Secondly, the possibility of publication bias has to be considered. The asymmetry as seen in the funnel plot suggests that this could be the case for small studies with no significant effect on any cardiovascular event with calcification. However, statistical tests did not indicate significant publication bias. Furthermore, there was significant heterogeneity among the studies that could largely be explained by the different investigational tools on the one hand and different duration of follow up on the other. For example, patients who are being evaluated by CT for coronary symptoms, run a substantially higher risk with a shorter time horizon than those in whom calcifications are found accidentally. When we analyzed the risk for any cardiovascular event separately in relation to the type of imaging technique and time of follow-up ( $<5$ years and $>5$ years), heterogeneity disappeared for the US and CT studies (Figure 4), but significant heterogeneity remained present in studies using plain radiography with follow-up longer than five years. This persistent heterogeneity can possibly be explained by differences in population characteristics and calcification. In the studies with diabetic patients medial calcification was present in most cases. Medial calcification is associated with higher odds ratios for events than intimal calcifications in diabetic patients and might thus be an explanation for persistent heterogeneity in radiographic studies with a long follow-up period. ${ }^{9}$ Also the range of follow up time in these populations was much greater $(2-26$ years in plain radiograph studies, 1.5-16 in US studies, and 2.7-6.9 in CT studies).

With respect to selection mechanisms and baseline risk, subgroup analysis of a renal insufficiency population showed the highest risk. CT data predicted the events best with the highest odds ratio. Moreover, when calcium was seen with coronary $\mathrm{CT}$, events occurred after a relatively short follow up period. Although coronary calcifications as detected by CT were associated with a greater risk of events than calcification in other areas, the difference in study populations preclude meaningful conclusions about the superiority of CT relative to other imaging modalities. Moreover, compared with plain X-rays or ultrasound studies, noncontrasted CT scans and EBCT are likely to be more sensitive for detecting small amounts of calcification. Interestingly, in hemodialysis patients were plain radiography or US were used to detect calcifications some of the highest odds ratios for all cause mortality and cardiovascular mortality were seen. ${ }^{19,28,30,33,36}$ This might reflect their lower sensitivity for detecting any calcification. Half of the studies that reported on all cause mortality concerned a high-risk patient group with renal insufficiency. This influenced the odds ratio resulting in a relatively high risk for all cause mortality. Nevertheless, when we calculated the odds ratio for populations without renal insufficiency, this was still significantly increased (3.43; $95 \%$ CI 1.26 to 9.32 ). 
The results of this meta-analysis help to clarify the importance of calcification in all sorts of patients. Although the reported odds ratios should be interpreted with caution because of substantial heterogeneity across studies, it showed that in almost any case of calcification there is an increased cardiovascular risk. It is important to realize that subjects with vascular calcification are high-risk patients. Currently, however, there is no specific therapy to reduce these calcifications and no evidence to support a better cardiovascular outcome with calcification reduction, although data from animal research and from human cells in vivo on the actions of matrix Gla protein as an active calcification inhibitor show promising results. ${ }^{40-42}$ The use of statins reduces the cardiovascular event rate in almost all patient groups, but data on their calcification inhibiting properties are conflicting. ${ }^{43-48}$

To our knowledge, there are no studies exploring the effect of treating all cardiovascular risk factors in patients based only on the presence of calcifications. However, extensive coronary calcifications justify further investigations for coronary plaques. Therefore, we feel that the practicing physician is also justified to screen subjects, in whom calcifications are accidentally detected, for treatable risk factors. If these are present, the cardiovascular risk according to risk charts should be assessed. If the outcome exceeds the threshold defined in guidelines, the physician should take measures to reduce the cardiovascular risk of his or her patient.

\section{Acknowledgments}

None of the authors had any conflict of interest to the subject of this manuscript. RJMW Rennenberg and AA Kroon had full access to all of the data in the study and take responsibility for the integrity of the data and the accuracy of the data analysis. There were no commercial funding sources involved.

\section{References}

1. Gidding SS, Bookstein LC, Chomka EV. Usefulness of electron beam tomography in adolescents and young adults with heterozygous familial hypercholesterolemia. Circulation. 1998;98:2580-2583.

2. Vliegenthart R, Hollander M, Breteler MMB, et al. Stroke is associated with coronary calcification as detected by electron-beam CT: The Rotterdam Coronary Calcification Study. Stroke. 2002;33:462-465.

3. Kondos GT, Hoff JA, Sevrukov A, et al. Electron-beam tomography coronary artery calcium and cardiac events: a 37-month follow-up of 5635 initially asymptomatic low- to intermediate-risk adults. Circulation. 2003;107:2571-2576.

4. Shaw LJ, Raggi P, Schisterman E, Berman DS, Callister TQ. Prognostic value of cardiac risk factors and coronary artery calcium screening for all-cause mortality. Radiology. 2003;228:826-833.

5. Iribarren C, Sidney S, Sternfeld B, Browner WS. Calcification of the aortic arch: risk factors and association with coronary heart disease, stroke, and peripheral vascular disease. JAMA. 2000;283: 2810-2815.
6. Iribarren C, Go AS, Tolstykh I, Sidney S, Johnston SC, Spring DB. Breast vascular calcification and risk of coronary heart disease, stroke, and heart failure. J Womens Health (Larchmt). 2004;13:381-389; discussion 390-392.

7. Hollander M, Hak AE, Koudstaal PJ, et al. Comparison between measures of atherosclerosis and risk of stroke: The Rotterdam Study. Stroke. 2003;34:2367-2372.

8. Kemmeren JM, van Noord PA, Beijerinck D, Fracheboud J, Banga JD, van der Graaf Y. Arterial calcification found on breast cancer screening mammograms and cardiovascular mortality in women: The DOM Project. Doorlopend Onderzoek Morbiditeit en Mortaliteit. Am J Epidemiol. 1998;147:333-341.

9. Niskanen L, Siitonen O, Suhonen M, Uusitupa MI. Medial artery calcification predicts cardiovascular mortality in patients with NIDDM. Diabetes Care. 1994;17:1252-1256.

10. Pletcher MJ, Tice JA, Pignone M, Browner WS. Using the coronary artery calcium score to predict coronary heart disease events: a systematic review and meta-analysis. Arch Intern Med. 2004;164:1285-1292.

11. Kemmeren JM, Beijerinck D, van Noord PA, et al. Breast arterial calcifications: association with diabetes mellitus and cardiovascular mortality. Work in progress. Radiology. 1996;201:75-78.

12. Becker A, Knez A, Becker C, et al. [Prediction of serious cardiovascular events by determining coronary artery calcification measured by multi-slice computed tomography]. Dtsch Med Wochenschr. 2005;130:2433-2438.

13. Becker A, Leber A, Becker C, Knez A. Predictive value of coronary calcifications for future cardiac events in asymptomatic individuals. Am Heart J. 2008;155:154-160.

14. Egger M, Smith GD, Altman DG. Systematic Reviews in Health Care: Meta-Analysis in Context. London: BMJ Publishing Group; 2001. p. 487.

15. LaMonte MJ, FitzGerald SJ, Church TS, et al. Coronary artery calcium score and coronary heart disease events in a large cohort of asymptomatic men and women. Am J Epidemiol. 2005;162:421-429.

16. Arad Y, Goodman KJ, Roth M, Newstein D, Guerci AD. Coronary calcification, coronary disease risk factors, C-reactive protein, and atherosclerotic cardiovascular disease events: the St. Francis Heart Study. J Am Coll Cardiol. 2005;46:158-165.

17. Budoff MJ, Shaw LJ, Liu ST, et al. Long-term prognosis associated with coronary calcification: observations from a registry of 25,253 patients. J Am Coll Cardiol. 2007;49:1860-1870.

18. Benjamin EJ, Plehn JF, D’Agostino RB, et al. Mitral annular calcification and the risk of stroke in an elderly cohort. $N$ Engl J Med. 1992;327:374-379.

19. Blacher J, Guerin AP, Pannier B, Marchais SJ, London GM. Arterial calcifications, arterial stiffness, and cardiovascular risk in end-stage renal disease. Hypertension. 2001;38:938-942.

20. Boon A, Lodder J, Cheriex E, Kessels F. Mitral annulus calcification is not an independent risk factor for stroke: a cohort study of 657 patients. J Neurol. 1997;244:535-541.

21. Everhart JE, Pettitt DJ, Knowler WC, Rose FA, Bennett PH. Medial arterial calcification and its association with mortality and complications of diabetes. Diabetologia. 1988;31:16-23.

22. Fox CS, Vasan RS, Parise H, et al. Mitral annular calcification predicts cardiovascular morbidity and mortality: the Framingham Heart Study. Circulation. 2003;107:1492-1496.

23. Fox E, Harkins D, Taylor H, et al. Epidemiology of mitral annular calcification and its predictive value for coronary events in African Americans: the Jackson Cohort of the Atherosclerotic Risk in Communities Study. Am Heart J. 2004;148:979-984.

24. Keelan PC, Bielak LF, Ashai K, et al. Long-term prognostic value of coronary calcification detected by electron-beam computed tomography in patients undergoing coronary angiography. Circulation. 2001;104:412-417.

25. Kitamura A, Iso H, Imano H, et al. Carotid intima-media thickness and plaque characteristics as a risk factor for stroke in Japanese elderly men. Stroke. 2004;35:2788-2794. 
26. Kizer JR, Wiebers DO, Whisnant JP, et al. Mitral annular calcification, aortic valve sclerosis, and incident stroke in adults free of clinical cardiovascular disease: the Strong Heart Study. Stroke. 2005;36:2533-2537.

27. Lehto S, Niskanen L, Suhonen M, Ronnemaa T, Laakso M. Medial artery calcification. A neglected harbinger of cardiovascular complications in non-insulin-dependent diabetes mellitus. Arterioscler Thromb Vasc Biol. 1996;16:978-983.

28. London GM, Guerin AP, Marchais SJ, Metivier F, Pannier B, Adda H. Arterial media calcification in end-stage renal disease: impact on all-cause and cardiovascular mortality. Nephrol Dial Transplant. 2003; 18:1731-1740

29. Raggi P, Callister TQ, Cooil B, et al. Identification of patients at increased risk of first unheralded acute myocardial infarction by electronbeam computed tomography. Circulation. 2000;101:850-855.

30. Salgueira M, del Toro N, Moreno-Alba R, Jimenez E, Areste N, Palma A. Vascular calcification in the uremic patient: a cardiovascular risk? Kidney Int Suppl. 2003:S119-S121.

31. Taylor AJ, Bindeman J, Feuerstein I, Cao F, Brazaitis M, O’Malley PG. Coronary calcium independently predicts incident premature coronary heart disease over measured cardiovascular risk factors: mean three-year outcomes in the Prospective Army Coronary Calcium (PACC) project. J Am Coll Cardiol. 2005;46:807-814.

32. Walsh CR, Cupples LA, Levy D, et al. Abdominal aortic calcific deposits are associated with increased risk for congestive heart failure: the Framingham Heart Study. Am Heart J. 2002;144:733-739.

33. Wang AY, Wang M, Woo J, et al. Cardiac valve calcification as an important predictor for all-cause mortality and cardiovascular mortality in long-term peritoneal dialysis patients: a prospective study. J Am Soc Nephrol. 2003;14:159-168.

34. Wong ND, Hsu JC, Detrano RC, Diamond G, Eisenberg H, Gardin JM. Coronary artery calcium evaluation by electron beam computed tomography and its relation to new cardiovascular events. Am J Cardiol. 2000;86:495-498.

35. Gardin JM, McClelland R, Kitzman D, et al. M-mode echocardiographic predictors of six- to seven-year incidence of coronary heart disease, stroke, congestive heart failure, and mortality in an elderly cohort (the Cardiovascular Health Study). Am J Cardiol. 2001;87:1051-1057.

36. Okuno S, Ishimura E, Kitatani K, et al. Presence of abdominal aortic calcification is significantly associated with all-cause and cardiovascular mortality in maintenance hemodialysis patients. Am J Kidney Dis. 2007;49:417-425.
37. Willens HJ, Chirinos JA, Schob A, Veerani A, Perez AJ, Chakko S. The relation between mitral annular calcification and mortality in patients undergoing diagnostic coronary angiography. Echocardiography. 2006;23:717-722.

38. Greenland P, LaBree L, Azen SP, Doherty TM, Detrano RC. Coronary artery calcium score combined with Framingham score for risk prediction in asymptomatic individuals. JAMA. 2004;291:210-215.

39. Boon A, Lodder J, Cheriex E, Kessels F. Risk of stroke in a cohort of 815 patients with calcification of the aortic valve with or without stenosis. Stroke. 1996;27:847-851.

40. Proudfoot D, Skepper JN, Shanahan CM, Weissberg PL. Calcification of human vascular cells in vitro is correlated with high levels of matrix Gla protein and low levels of osteopontin expression. Arterioscler Thromb Vasc Biol. 1998;18:379-388.

41. Luo G, Ducy P, McKee MD, et al. Spontaneous calcification of arteries and cartilage in mice lacking matrix GLA protein. Nature. 1997;386:78-81

42. Schurgers LJ, Teunissen KJ, Knapen MH, et al. Novel conformationspecific antibodies against matrix gamma-carboxyglutamic acid (Gla) protein: undercarboxylated matrix Gla protein as marker for vascular calcification. Arterioscler Thromb Vasc Biol. 2005;25:1629-1633.

43. Kizu A, Shioi A, Jono S, Koyama H, Okuno Y, Nishizawa Y. Statins inhibit in vitro calcification of human vascular smooth muscle cells induced by inflammatory mediators. J Cell Biochem. 2004;93:1011-1019.

44. Budoff MJ, Yu D, Nasir K, et al. Diabetes and progression of coronary calcium under the influence of statin therapy. Am Heart J. 2005;149:695-700.

45. Cowell SJ, Newby DE, Prescott RJ, et al. A randomized trial of intensive lipid-lowering therapy in calcific aortic stenosis. $N$ Engl J Med. 2005;352:2389-2397.

46. Schmermund A, Achenbach S, Budde T, et al. Effect of intensive versus standard lipid-lowering treatment with atorvastatin on the progression of calcified coronary atherosclerosis over 12 months: a multicenter, randomized, double-blind trial. Circulation. 2006;113:427-437.

47. Arad Y, Spadaro LA, Roth M, Newstein D, Guerci AD. Treatment of asymptomatic adults with elevated coronary calcium scores with atorvastatin, vitamin C, and vitamin E: the St. Francis Heart Study randomized clinical trial. J Am Coll Cardiol. 2005;46:166-172.

48. Houslay ES, Cowell SJ, Prescott RJ, et al. Progressive coronary calcification despite intensive lipid-lowering treatment: a randomised controlled trial. Heart. 2006;92:1207-1212. 
